\title{
Stage IB2 Cervical Cancer AJCC v6 and v7
}

National Cancer Institute

\section{Source}

National Cancer Institute. Stage IB2 Cervical Cancer A/CC v6 and v7. NCI Thesaurus.

Code C6318.

Stage IB2 includes: T1b2, N0, M0. T1b2: Clinically visible lesion more than $4.0 \mathrm{~cm}$ in greatest dimension. N0: No regional lymph node metastasis. M0: No distant metastasis. (AJCC 6th and 7th eds.) 